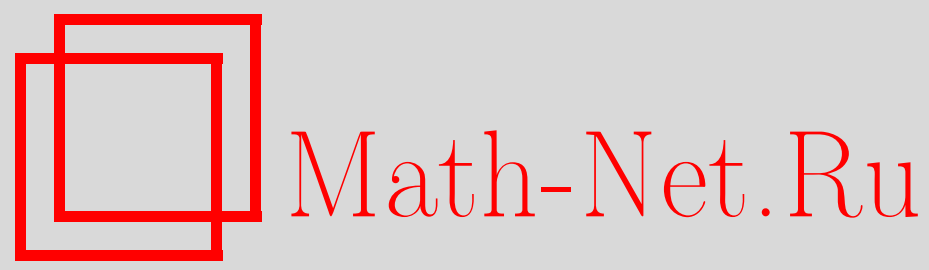

А. Г. Токарев, Контрпримеры к формуле Троттера в локально выпуклых пространствах, Матем. заметки, 1996, том 59, выпуск 6, 947-950

DOI: https://doi.org/10.4213/mzm1797

Использование Общероссийского математического портала MathNet.Ru подразумевает, что вы прочитали и согласны с пользовательским соглашением

http://www. mathnet.ru/rus/agreement

Параметры загрузки:

IP : 35.173 .219 .12

26 апреля 2023 г., 13:37:35

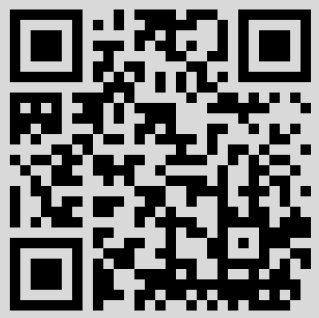




\section{КОНТРПРИМЕРЫ К ФОРМУЛЕ ТРОТТЕРА В ЛОКАЛЬНО ВЫПУКЛЫХ ПРОСТРАНСТВАХ}

\section{А. Г. Токарев}

Определения и терминология. Всюду ниже $\mathbb{N}=\{1,2, \ldots\} ; \mathbb{R}^{\infty}$-счетное произведение прямых; $\mathbb{R}^{(\infty)}$ - векторное пространство финитных последовательностей вещественных чисел; $\left\{e_{i}\right\}_{i=1}^{\infty}-$ стандартный базис в $\mathbb{R}^{(\infty)} ;\|x\|=\left|x_{1}\right|+$ $\cdots+\left|x_{n}\right|$ - норма в $\mathbb{R}^{n}, n \in \mathbb{N} ; J_{m}$ означает $m \times m$-матрицу, у которой под главной диагональю единицы, а остальные элементы равны нулю, $J_{m}^{T}$ транспонирована к $J_{m} ; L_{1} \oplus L_{2}$ - сумма локально выпуклых пространств $L_{1}$ и $L_{2}$, а $L_{1} \times L_{2}$ - их произведение.

Если $E$ - локально выпуклое пространство (ЛВП), то $\mathscr{L}(E)$ - пространство непрерывных линейных отображений из $E$ в $E$ с топологией поточечной сходимости, в $\mathscr{L}\left(\mathbb{R}^{n}\right)$ введена норма $\|\cdot\|$, индуцированная нормой из $\mathbb{R}^{n}, n \in \mathbb{N} ;\left\langle v_{\alpha} \mid \alpha \in I\right\rangle$ подпространство в $E$, порожденное векторами $\left\{v_{\alpha}\right\}_{\alpha \in I} ; \bar{A}$ - непрерывное линейное продолжение $A \in \mathscr{L}(E)$ на пополнение $E$.

Если $A_{n}(n=1,2, \ldots)$ - семейство конечномерных линейных отображений $A_{n} \in \mathscr{L}\left(\mathbb{R}^{k_{n}}\right)$, то $D\left(A_{n}\right)=\left\langle e_{i} \mid s_{n-1}<i \leqslant s_{n}\right\rangle$, где $s_{n}=\sum_{i=1}^{n} k_{i}, s_{0}=0$. Будем отождествлять $D\left(A_{n}\right)$ и $\mathbb{R}^{k_{n}}$ посредством отображения, ставящего вектору $e_{s_{n-1}+i}, 1 \leqslant i \leqslant k_{n}$, в соответствие орт в $\mathbb{R}^{k_{n}}$ с единицей на $i$-м месте (это дает возможность считать, что каждый $A_{n}$ определен также и на $\left.D\left(A_{n}\right)\right)$. При этом базис $e_{s_{n-1}+i}, 1 \leqslant i \leqslant k_{n}$, в $D\left(A_{n}\right)$ позволяет определять $A_{n}$ с помощью матрицы.

Прямой суммой конечномерных операторов $A_{n}(n=1,2, \ldots)$ назовем линейное отображение $A=\bigoplus_{n=1}^{\infty} A_{n}$ из $\mathbb{R}^{(\infty)}$ в $\mathbb{R}^{(\infty)}$, которое совпадает с $A_{n}$ при сужении на $D\left(A_{n}\right) ;$ положим $\exp (A)=\bigoplus_{n=1}^{\infty} \exp \left(A_{n}\right)$.

Обозначим через $S_{m}, m \in \mathbb{N}$, множество прямых сумм $A=\bigoplus_{i=1}^{\infty} A_{i}, A_{i} \in$ $\mathscr{L}\left(\mathbb{R}^{k_{i}}\right)$, таких, что $C(A)=\sup _{i}\left\|A_{i}\right\|<\infty$ и $k_{i} \leqslant m$ для всех $i \in \mathbb{N}$.

Если $E$ - ЛВП, то аналогично [1], будем обозначать символом unex $(E)$ множество отображений $A \in \mathscr{L}(E)$, таких что задача Коши для уравнения

$$
\dot{x}=A x, \quad x(0)=x_{0}
$$

однозначно разрешима на полупрямой $t \geqslant 0$ для всякого $x_{0} \in E$. Например, $\mathscr{L}\left(\mathbb{R}^{n}\right) \in \operatorname{unex}\left(\mathbb{R}^{n}\right), n \in \mathbb{N}$.

Если $A \in \operatorname{unex}(E)$ и $G_{A}\left(t, x_{0}\right)$ значение решения (1) в точке $t \geqslant 0$, то определим отображение $\exp (t A)$ из $E$ в $E$ так: $\exp (t A) x=G_{A}(t, x), x \in E$.

Будем говорить, что в ЛВП $E$ справедлива формула Троттера (называемая также формулой Далецкого-Троттера), если каковы бы ни были непрерывные отображения $A, B$ из $E$ в $E$, такие что $A, B, A+B \in \operatorname{unex}(E)$, справедливо равенство

$$
\lim _{n \rightarrow \infty}\left(e^{\frac{t}{n} A} e^{\frac{t}{n} B}\right)^{n} x=e^{t(A+B)} x
$$

для всех $x \in E$ (cp. [2]).

Предварительные результаты. Сформулируем и докажем две леммы. 
ЛЕмма 1. Пусть $m \in \mathbb{N}$ u топология в $\mathbb{R}^{(\infty)}$ задана семейством преднорм $\left\{p_{\alpha}\right\}_{\alpha \in I}$, так что для всякого $\alpha \in I$ существует $\beta \in I$, такое что для л1обого $A=\bigoplus_{i=1}^{\infty} A_{i} \in S_{m}$

$$
p_{\alpha}(A x) \leqslant C(A) p_{\beta}(x), \quad x \in \mathbb{R}^{(\infty)} .
$$

Тогда $S_{m} \subset \mathscr{L}\left(\mathbb{R}^{(\infty)}\right)$ и если $A=\bigoplus_{i=1}^{\infty} A_{i} \in S_{m}$, mo $\bar{A} \in \operatorname{unex}(E)$ u $\exp (t \bar{A})=\overline{\exp (t A)}$, әде $E$ пополнение $\mathbb{R}^{(\infty)}$.

ДокАЗАТЕЛЬСТво. То, что $S_{m} \subset \mathscr{L}\left(\mathbb{R}^{(\infty)}\right)$ очевидно.

Для всех $t \geqslant 0$ положим $x(t)=\overline{\exp (t A)} x_{0}, x(0)=x_{0}$. Убедимся, что $x(\cdot)-$ решение (1) для $\bar{A}$. Очевидно, это так, когда $x_{0} \in \mathbb{R}^{(\infty)}$.

Если $x_{0}^{k} \rightarrow x_{0}$ при $k \rightarrow \infty$, где $x_{0}^{k} \in \mathbb{R}^{(\infty)}, x_{0} \in E$, то $x^{k}(t) \rightarrow x(t)$ при $k \rightarrow \infty$, где $x_{0}^{k}(t)=\overline{\exp (t A)} x_{0}^{k}$, так как $\overline{\exp (t A)} \in \mathscr{L}(E)$.

Покажем, что

$$
\lim _{h \rightarrow 0} \lim _{k \rightarrow \infty} \frac{x^{k}(t+h)-x^{k}(t)}{h}=\lim _{k \rightarrow \infty} \lim _{h \rightarrow 0} \frac{x^{k}(t+h)-x^{k}(t)}{h} .
$$

Для этого достаточно доказать (см. [3, с. 39-42]), что для всякого $\alpha \in I$

$$
\begin{aligned}
p_{\alpha}\left(\frac{x^{k}(t+h)-x^{k}(t)}{h}-\dot{x}^{k}(t)\right) & =p_{\alpha}\left(\left(\frac{e^{h A}-1}{h}-A\right) e^{t A} x_{0}^{k}\right) \\
& \leqslant e^{t C(A)} p_{\beta}\left(x_{0}^{k}\right) \sup _{i}\left\|\frac{e^{h A_{i}}-1}{h}-A_{i}\right\| \rightarrow 0
\end{aligned}
$$

при $h \rightarrow 0$ равномерно по $k=1,2, \ldots$, но это так, поскольку $\left\{p_{\beta}\left(x_{0}^{k}\right)\right\}_{k=1}^{\infty}$ ограничено, а $A_{i} \in \mathscr{L}\left(\mathbb{R}^{k_{i}}\right), k_{i} \leqslant m$, и $\left\|A_{i}\right\| \leqslant C, i=1,2, \ldots$.

В итоге

$$
\lim _{h \rightarrow 0} \frac{x(t+h)-x(t)}{h}=\lim _{k \rightarrow \infty} \dot{x}^{k}(t)=\bar{A} x(t) .
$$

Если $\operatorname{pr}_{i}-$ проектор в $E$ на $D\left(A_{i}\right)$ вдоль $D\left(A_{j}\right), j \neq i$, то $\operatorname{pr}_{i}(x(\cdot))$ решение (1) для $\overline{A_{i}}$ с данными Коши $\operatorname{pr}_{i}\left(x_{0}\right)$, когда $x(\cdot)$ - решение $(1)$ для $\bar{A}$ и $x(0)=x_{0}$, поскольку $\bar{A}\left(D\left(A_{j}\right)\right) \subset D\left(A_{j}\right), j \in \mathbb{N}$. Это влечет единственность. Лемма доказана.

Определим в $\mathbb{R}^{(\infty)}$ отображения $A_{0}, B_{0}, C_{0}$ следующим образом: $A_{0}=\bigoplus_{i=1}^{\infty} A_{i}$; $B_{0}=\bigoplus_{i=1}^{\infty} B_{i}$, где $A_{2 i}=-J_{2} ; A_{2 i+1}=J_{2}(i=1,2, \ldots), A_{1}$ - нулевая матрица размерности $1, B_{i}=J_{4}(i=1,2, \ldots) ; C_{0}=A_{0}+B_{0}$. Ясно, что $A_{0}, B_{0}, C_{0} \in S_{4}$.

Лемма 2. Для любого $n \in \mathbb{N} u$ всех $t>0$

$$
v_{n}=\left(e^{\frac{t}{n} A_{0}} e^{\frac{t}{n} B_{0}}\right)^{n} e_{1} \in U_{4 n}+\frac{t^{4 n}}{n^{4 n}} \frac{1}{3 !} e_{4 n+1},
$$

əде $U_{m}=\left\langle e_{i} \mid 1 \leqslant i \leqslant m\right\rangle$.

ДоКАЗАТЕЛЬСТво. Используя Явный вид $\exp \left(J_{m}\right)$ и инвариантность $U_{3}$ относительно $A_{0}$, имеем

$$
e^{\frac{t}{n} A_{0}} e^{\frac{t}{n} B_{0}} e_{1} \in e^{\frac{t}{n} A_{0}}\left(U_{3}+\frac{t^{3}}{n^{3}} \frac{1}{3 !} e_{4}\right) \subset U_{4}+\frac{t^{4}}{n^{4}} \frac{1}{3 !} e_{5} .
$$

Повторяя те же рассуждения еще $n-1$ раз придем к необходимому результату. Лемма доказана. 


\section{Бесконечные топологические суммы.}

ПримеР 1. Обозначим через $E_{1}$ векторное пространство $\mathbb{R}^{(\infty)}$, наделенное топологией суммы подпространств, порожденных векторами $e_{i}$, $i=1,2, \ldots$. Тогда $A_{0}, B_{0}, C_{0}=A_{0}+B_{0} \in \operatorname{unex}\left(E_{1}\right)$, но не удовлетворяют формуле Троттера.

ДоКАЗАТЕЛЬСтво. То, что $A_{0}, B_{0}, C_{0} \in u n e x\left(E_{1}\right)$ вытекает из леммы 1 , так как они удовлетворяют ее условиям.

Из леммы 2 следует, что левая часть (2) расходится в точке $e_{1}$ поскольку $v_{n}$ $(n=1,2, \ldots)$ не образуют последовательность Коши в $E_{1}$.

СледСТвИЕ 1. Для любой бесконечной топологической суммы $Е$ локально выпуклых пространств найдутся отобрахсения, удовлетворяющие утверждению примера 1.

ДокаЗАТЕльство. Представим $E$ в виде $E=L \bigoplus E_{0}$, где $L$ содержит счетное число слагаемых $L_{i}(i=1,2, \ldots)$, входящих в $E$, а $E_{0}$ - все остальные. Раскладывая каждое $L_{i}$ в сумму $L_{i}=L_{i}^{1} \oplus L_{i}^{2}$, где размерность $L_{i}^{1}$ равна 1 , и пользуясь тем, что $\bigoplus_{i=1}^{\infty} L_{i}^{1}=E_{1}$, получим $E=E_{1} \oplus L^{2} \bigoplus E_{0}$, где $L^{2}=\bigoplus_{i=1}^{\infty} L_{i}^{2}$.

Продолжая отображения $A_{0}, B_{0}$ и $C_{0}$ из примера 1 нулем на $L^{2} \oplus E_{0}$, получим нужные примеры.

Пространства Фреше. Теперь определим в $\mathbb{R}^{(\infty)}$ топологию с помощью семейства норм

$$
p_{k}(x)=\sum_{n=1}^{\infty}\left|x_{n}\right| e^{(n+k)^{2}}, \quad k=1,2, \ldots ; \quad x=\sum_{n=1}^{\infty} x_{n} e_{n} .
$$

Проверим, что оно удовлетворяет условиям леммы 1.

Если $T=\bigoplus_{i=1}^{\infty} T_{i} \in S_{m}, m \in \mathbb{N}$, то через $\operatorname{pr}_{i}$ обозначим проектор в $\mathbb{R}^{\infty}$ на $D\left(T_{i}\right)$, вдоль $D\left(T_{j}\right), j \neq i$, определим последовательность $\left\{s_{i}\right\}_{i=1}^{\infty}$ условием $D\left(T_{i}\right)=\left\langle e_{n} \mid s_{i-1}<n \leqslant s_{i}\right\rangle, s_{0}=0$, имеем $s_{i}-s_{i-1} \leqslant m$.

Положим $y=T x$, тогда

$$
\begin{aligned}
p_{k}(y) & \leqslant \sum_{i=1}^{\infty}\left\|\operatorname{pr}_{i}(y)\right\| e^{\left(s_{i}+k\right)^{2}} \leqslant \sum_{i=1}^{\infty}\left\|T_{i} \operatorname{pr}_{i}(x)\right\| e^{\left(s_{i}+k\right)^{2}} \\
& \leqslant C(T) \sum_{i=1}^{\infty}\left\|\operatorname{pr}_{i}(x)\right\| e^{\left(s_{i}+k\right)^{2}} \leqslant C(T) p_{k+m}(x) .
\end{aligned}
$$

ПримеР 2. Пусть $F$ - пополнение $\mathbb{R}^{(\infty)}$ в топологии, описанной выше. Тогда $F$ - ядерное пространство Фреше с непрерывной нормой (см. [1, с. 102]) $и \overline{A_{0}}, \overline{B_{0}}, \overline{C_{0}}=\overline{A_{0}}+\overline{B_{0}} \in \operatorname{unex}(F)$ но для них не справедлива формула Tpommepa.

ДоКАЗАТЕЛЬСТВо. То, что $\overline{A_{0}}, \overline{B_{0}}, \overline{C_{0}} \in \operatorname{unex}(F)$ следует из леммы 1.

Чтобы доказать вторую часть утверждения покажем, что последовательность $v_{n}(n=1,2, \ldots)$ из леммы 2 расходится в $F$ при $t>0$.

Действительно, в силу того, что

$$
v_{n} \in U_{4 n}+\frac{t^{4 n}}{n^{4 n}} \cdot \frac{1}{(3 !)^{n}} e_{4 n+1}
$$

и так как

$$
\ln \left[\frac{t^{4 n}}{n^{4 n}} \cdot \frac{1}{(3 !)^{n}}\right] \geqslant-M n-N n^{3 / 2},
$$


где $M, N \in \mathbb{R}$ - зависящие от $t>0$ постоянные, имеем

$$
p_{k}\left(v_{n}\right) \geqslant p_{k}\left(\frac{t^{4 n}}{n^{4 n}} \cdot \frac{1}{(3 !)^{n}} e_{4 n+1}\right) \geqslant e^{-n(M+N \sqrt{n})+(4 n+1+k)^{2}} .
$$

И, значит, $\left\{v_{n}\right\}_{n=1}^{\infty}$ не фундаментальна в $F$, так как $p_{k}\left(v_{n}\right) \rightarrow \infty$ при $n \rightarrow \infty$.

Положим $A=\bigoplus_{i=1}^{\infty} A_{i}, B=\bigoplus_{i=1}^{\infty} B_{i}$, где $A_{i}=J_{4}^{T}(i=1,2, \ldots) ; B_{1}=0$, $D\left(B_{1}\right)=\left\langle e_{1}\right\rangle, B_{2 i}=-J_{2}^{T}, B_{2 i+1}=J_{2}^{T}(i=1,2, \ldots) ; C=A+B$. Имеем $A, B, C \in S_{4}$.

ПРИМЕР 3. Поскольку $\mathbb{R}^{(\infty)}$ в топологии покоординатной сходимости удовлетворяет условиям леммы 1 и его пополнением является $\mathbb{R}^{\infty}$, то для отобрахсений определенных выше имеем $\bar{A}, \bar{B}, \bar{C} \in \operatorname{unex}\left(\mathbb{R}^{\infty}\right)$, но $\bar{A}$ и $\bar{B}$ не удовлетворяют формуле Троттера.

ДоКАЗАТЕЛЬСтво. Положим $x_{0}=\left(a_{1}, \ldots\right), w_{n}=\left(\exp \left(\frac{t}{n} \bar{A}\right) \exp \left(\frac{t}{n} \bar{B}\right)\right)^{n} x_{0}$, $w_{n}=\left(w_{n}^{1}, w_{n}^{2}, \ldots\right)$. Прямые вычисления с использованием явного вида $\exp \left(\frac{t}{n} J_{m}^{T}\right)$ дают $w_{n}^{1}=\lambda_{1} a_{1}+\cdots+\lambda_{4 n+1} a_{4 n+1}$, где $\lambda_{4 n+1} \neq 0$. Поэтому выбирая $a_{4 n+1}$ так, чтобы $w_{n}^{1} \rightarrow \infty$ при $n \rightarrow \infty$, получим, что левая часть (2) расходится в точке $x_{0}$.

СлЕДСТВИЕ 2. Для всякого бесконечного произведения $E$ локально выпуклых пространств существуют отобрахсения, для которых формула Троттера не справедлива.

ДокАЗАТЕльство. Представим $E$ в виде $E=L \times E_{0}$, где $L$ содержит счетное число множителей $L_{i}, i=1,2, \ldots$, входящих в $E$, а $E_{0}$ - остальные. Раскладывая каждое $L_{i}$ в сумму $L_{i}=L_{i}^{1} \bigoplus L_{i}^{2}$, где размерность $L_{i}$ равна 1 , и пользуясь тем, что $\times_{i=1}^{\infty} L_{i}^{1}=\mathbb{R}^{\infty}$, получим $E=\mathbb{R}^{\infty} \times L^{2} \times E_{0}$, где $L^{2}=\times_{i=1}^{\infty} L_{i}^{2}$.

Продолжая отображения из примера 3 нулем на $L^{2} \times E_{0}$, получим нужные примеры.

В заключение отметим, что существуют неметризуемые локально выпуклые пространства, в которых формула Троттера справедлива.

ПрИмер 4. Пусть $B$ - бесконечномерное банахово пространство $и \boldsymbol{B}_{\sigma}$ обозначает векторное пространство $B$, наделенное слабой топологией. Поскольку $\mathscr{L}\left(B_{\sigma}\right)=\mathscr{L}(B)$ (см. [4, с. 201]) и формула Троттера справедлива в банаховых пространствах [2], она будет справедлива и в $\boldsymbol{B}_{\sigma}$.

Автор благодарит О.Г. Смолянова за постановку задачи и осуществление научного руководства этой работой.

Московский государственный университет

Поступило им. М.В. Ломоносова

04.10 .95

\section{СПИСОК ЦИТИРОВАННОЙ ЛИТЕРАТУРЫ}

1. Лобанов С. Г., Смолянов О. Г. // УМН. 1994. Т. 49. № 3. С. 93-168. 2. Chernoff P. R. // J. Funct. Anal. 1968. V. 2. P. 238-242. 3. Смолянов О. Г. Анализ на топологических линейных пространствах и его приложения. М.: Изд-во МГУ, 1979. 4. Шефер Х. Топологические векторные пространства. М.: Мир, 1971. 